\title{
Rectal obstruction after a vaginal posterior compartment polypropylene mesh fixed to the sacrospinous ligaments
}

\author{
Mark E. Vierhout • Mariella I. J. Withagen • \\ Jurgen J. Fütterer
}

Received: 27 October 2010 / Accepted: 30 January 2011 /Published online: 1 March 2011

(C) The Author(s) 2011. This article is published with open access at Springerlink.com

\begin{abstract}
We present a case in which a polypropylene mesh was placed over the posterior vaginal wall and was fixed to the sacrospinous ligaments on both sides. Postoperative a rectal obstruction developed which was only resolved after splitting the entire mesh in the midline. It is hypothesised that the obstruction was due to the fixation of the mesh with irresolvable suture material to the sacrospinous ligaments acting as a hinge on which the bowel folded.
\end{abstract}

Keywords Mesh · Complication · Surgery · Rectocele · Obstruction

\section{Introduction}

Vaginal meshes are widely used for the correction of pelvic organ prolapse (POP). They are however not without complications [1-5].

\section{Case report}

A 73-year-old para four healthy patient was referred to our institution because of recurrent pelvic organ prolapse

M. E. Vierhout $(\square) \cdot$ M. I. J. Withagen

Department of Obstetrics and Gynaecology,

Radboud University Nijmegen Medical Centre,

PO Box 9101, 6500 HB, Nijmegen, The Netherlands

e-mail: M.Vierhout@obgyn.umcn.nl

\section{J. J. Fütterer}

Department of Radiology,

Radboud University Nijmegen Medical Centre,

Nijmegen, The Netherlands
(POP). Previously, she underwent an uneventful vaginal hysterectomy with anterior and posterior repair but within 2 years a symptomatic recurrent POP developed and she was referred to our clinic. She had also undergone an earlier inguinal hernia operation.

On examination, we saw a POP stage 2 posterior wall prolapse with point $\mathrm{Bp}$ at the hymen $(\mathrm{Bp}=0)$. There was no significant anterior wall or vault prolapse.

To differentiate between a rectocele and a combined recto/enterocele a dynamic MRI was performed (Fig. 1) which revealed an isolated rectocele only.

She underwent vaginal repair operation in which the whole posterior wall was opened and an Ultrapro ${ }^{\mathrm{TM}}$ mesh was placed over the entire length of the rectocele. The Ultrapro $^{\mathrm{TM}}$ mesh was fixed bilateral to the sacrospinous ligaments with a single prolene 2-0 stitch with the help of a Capio ${ }^{\mathrm{TM}}$ device. After the placement the rectum was digitally checked for obstruction which was judged not to be the case. The vaginal wall was closed over the mesh without any removal of vaginal tissue.

Initially, she had an uneventful recovery but soon she started complaining of severe constipation for which she was readmitted 9 days after surgery. After treatment with laxatives, she had several bowel movements of watery thin stools and was discharged with laxatives. During admission, a rectal examination was performed and again no evidence of rectal obstruction was found; the mesh was palpable through the rectum but only loosely impressed the rectum with easy passage of the index finger. After discharge however, she kept complaining of extremely difficult bowel movements only possible after high doses of laxatives and producing only watery stool.

It was decided to perform a new dynamic MRI (Fig. 2) which revealed a clear rectal obstruction at around 


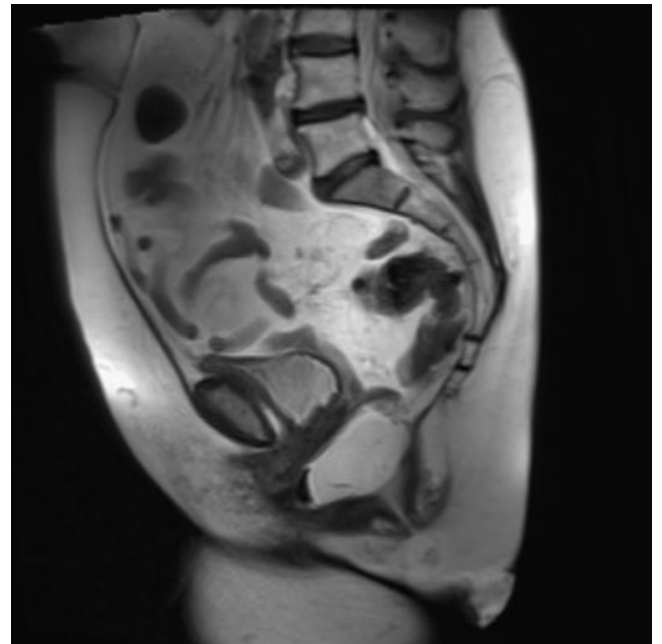

Fig. 1 Pre-operative midsagittal MRI during Valsalva showing a stage 2 anterior rectocele

$10 \mathrm{~cm}$ from the anus and a reoperation to release this stenosis was planned.

During the vaginal procedure, the posterior vaginal wall was reopened and the mesh was split in the middle over the entire length. No mesh was removed. Rectal digital examination and gentle manipulation now assured that the rectal canal was totally free. Almost directly after this second operation, she resumed her normal bowel movement pattern without any complaints. Her posterior wall prolapse had not recurred at 6 months after the reintervention.

\section{Discussion}

In this patient, an obstruction of the rectum occurred after placement of a vaginal polypropylene mesh which was fixed to the sacrospinous ligaments with irresolvable suturing material. In the recent literature, a number of studies regarding complications of vaginal mesh surgery have emerged [1-5] Complications specifically regarding the rectum are perforation during surgery [2], rectovaginal fistula [2], and transanal expulsion of a posterior mesh [2]. Lower bowel obstruction has, to our best knowledge, never been described as a complication of mesh implantation on the posterior vaginal wall.

\section{Points of specific interest}

The mesh was fixed with irresolvable suture material to the sacrospinous ligament. It is questionable however whether fixation should be done with irresolvable suture material. It has been shown that loosely woven polypropylene mesh show a rapidly ingrowth of connective tissue which makes fixation after this time unnecessary and possibly even harmful. In our case, possibly the obstruction could have been loosened by simple digital manipulation if resolvable suture material would have been used after the time needed to resolve the sutures in the sacrospinous ligament. However, this would probably not have altered the situation during her readmission at day 9 since also resolvable sutures than still have significant strength.

In our patient, the time between the operation and reintervention was long because the diagnosis of obstruction was wrongly rejected on the basis of physical examination. Only after making the second dynamic MRI did the diagnosis became evident. Apparently, it is difficult to diagnose this type of obstruction during physical examination. Possibly the obstruction was too high to be reached by the index finger or the mesh did not create a luminal obstruction but merely acted as a hinge on which the bowel folded and thus created a functional obstruction. One has to realise also that even a relatively mild reduction in the diameter of the rectum can significantly contribute to a functional obstruction of solid stool. Another explanation for the obstruction could involve the fact that the mesh makes the rectal wall more rigid and thus interfering with a normal downward movement of the rectum with limitation of the opening of the anorectal angle during defecation.

In conclusion, in this patient, a rectal obstruction occurred after mesh implantation for a posterior compartment prolapse. It was only diagnosed after making a dynamic MRI. The obstruction was solved by splitting the mesh in the midline.

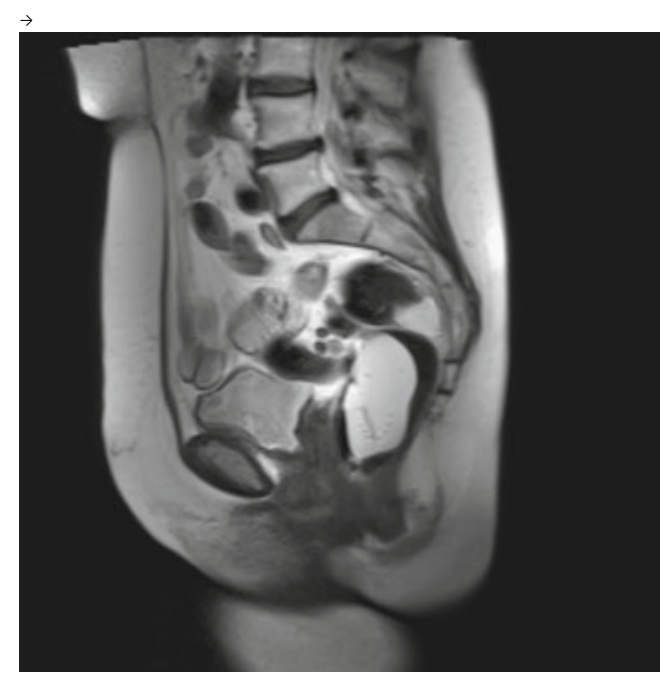

Fig. 2 Post-operative midsagittal MRI during Valsalva showing a rectal obstruction around $10 \mathrm{~cm}$ from the anus (rectum was filled with ultrasound gel) 


\section{Conflicts of interest None.}

Open Access This article is distributed under the terms of the Creative Commons Attribution Noncommercial License which permits any noncommercial use, distribution, and reproduction in any medium, provided the original author(s) and source are credited.

\section{References}

1. Jacquetin B, Cosson M (2009) Complications of vaginal mesh: our experience. Int Urogynecol J 20:893-896
2. Huffaker RK, Shull BL, Thomas JS (2009) A serious complication following placement of posterior prolift. Int Urogynecol J 20:1383-1385

3. Blandon RE, Gebhart JB, Trabuco EC, Clingele CJ (2009) Complications from vaginally placed mesh in reconstructive surgery. Int Urogynecol J 20:523-531

4. Bako A, Dhar R (2009) Review of synthetic mesh-related complications in pelvic floor reconstructive surgery. Int Urogynecol J 20:103-111

5. Margulies RU, Lewicky-Gaup C, Fenner DE, McGuire EJ, Clemens Q, DeLancey JOL (2008) Complications requiring reoperation following vaginal mesh kit procedures for prolapse. Am J Obstet Gynecol 199:678e1-678e4 